\title{
HAMARTOMA FOLÍCULO-SEBÁCEO CÍSTICO SIMULANDO NÓDULO MAMÁRIO: RELATO DE CASO*
}

\author{
Flávio Augusto Ataliba Caldas ${ }^{1}$, William Silva Neves ${ }^{1}$, Jorge Antônio Thomé ${ }^{2}$, \\ Eliane Rosin Cassiano Martinez ${ }^{3}$, Luciana Martins Tajara ${ }^{3}$
}

\begin{abstract}
Resumo 0 presente artigo tem por objetivo relatar um caso de um paciente do sexo masculino, de 35 anos de idade, apresentando tumoração na mama esquerda. Após estudo pelos métodos de imagens habituais o paciente foi submetido a biópsia, que revelou o resultado de hamartoma folículo-sebáceo cístico. Os autores apresentam breve revisão da literatura científica sobre essa rara lesão na mama masculina e seu diagnóstico diferencial com lesões malignas.

Unitermos: Hamartoma folículo-sebáceo cístico; Mama; Masculino; Revisão.
\end{abstract}

Abstract Folliculosebaceous cystic hamartoma simulating breast nodule: a case report.

We report a case of 35 year-old male patient presenting with a mass in the left breast. After conventional imaging examinations the patient was submitted to a biopsy, which showed folliculosebaceous cystic hamartoma. The authors present a brief review of the literature on this rare lesion in the male breast and its differential diagnosis with malignant masses.

Key words: Folliculosebaceous cystic hamartoma; Breast; Male; Review.

\section{INTRODUÇÃO}

Hamartoma folículo-sebáceo cístico (HFC) é uma rara lesão cutânea de elementos mesenquimais, sebáceos e foliculares ${ }^{(\mathbf{1})}$.

Os primeiros casos de HFC foram descritos em 1991 por Kimura et al. ${ }^{(2)}$, que determinaram os critérios clínicos e histopatológicos básicos desta lesão até então inédita na literatura.

Até o momento foram relatados 17 casos de HFC, sendo que todos eram extraepiteliais e se apresentavam como lesões grosseiramente verrucosas ou pedunculadas. Nosso relato traz um novo molde de apresentação dessa lesão incomum, desta vez simulando nódulo mamário.

\section{RELATO DO CASO}

Paciente do sexo masculino, 35 anos de idade, branco, procurou este serviço para

* Trabalho realizado no Instituto de Radiodiagnóstico Rio Preto S/C Ltda - Ultra-X, São José do Rio Preto, SP.

1. Médicos Residentes de Radiologia e Diagnóstico por Imagem do Ultra-X.

2. Médico Patologista do Hospital de Base da Faculdade de Medicina de São José do Rio Preto (Famerp).

3. Médicas Radiologistas do Ultra-X.

Endereço para correspondência: Dr. Flávio Augusto Ataliba Caldas. Rua Benjamim Constant, 3334, ap. 75, Vila Imperial. São José do Rio Preto, SP, 15015-600. E-mail: flaviocaldas@ hotmail.com

Recebido para publicação em 28/10/2004. Aceito, após revisão, em 16/2/2005. realização de mamografia e ultra-sonografia, com queixa de nodulação indolor há oito meses na mama esquerda evoluindo para leve incômodo nos últimos três meses. Negava doenças de base e uso de medicação. Ao exame físico foram observados assimetria mamária (sendo maior a mama esquerda) e nódulo palpável firme, móvel e indolor no quadrante superior medial da mama esquerda, sem derrame papilar ou qualquer alteração cutânea associada.

Na mamografia em médio-lateral a $90^{\circ}$ foi visualizado nódulo de média radioden-

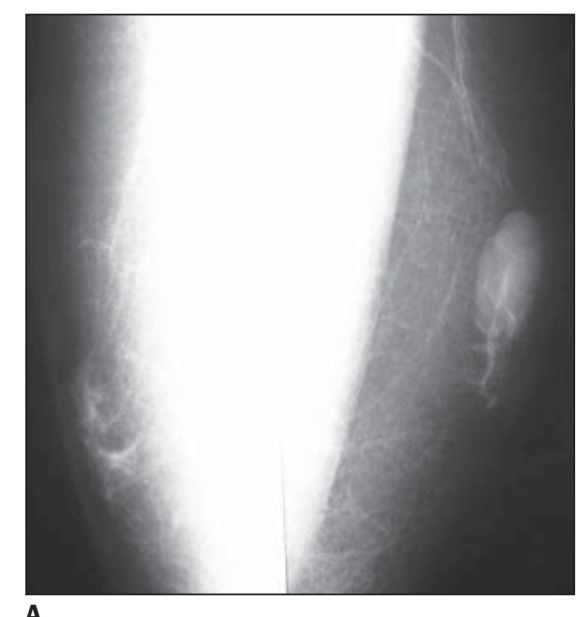

A

Figura 1. A: Radiografias obtidas em médio-lateral a $90^{\circ}$ mostram nódulo de média radiodensidade, contornos lobulados e bem definidos, medindo cerca de $2,5 \mathrm{~cm}$, localizado na região retroareolar da mama esquerda. B: Detalhe do nódulo descrito. sidade e com contornos lobulados, de localização retroareolar esquerda. O nódulo tinha aproximadamente $2,5 \mathrm{~cm}$ em seu maior eixo. Não foram observadas ginecomastia ou outras alterações mamárias associadas (Figura 1).

A ultra-sonografia revelou que a lesão retroareolar vista na mamografia tinha representatividade ecográfica como nódulo hipoecogênico e de contornos macrolobulados. A lesão apresentava fina cápsula ecogênica e tênue reforço acústico posterior, medindo cerca de 2,6 cm (Figura 2).

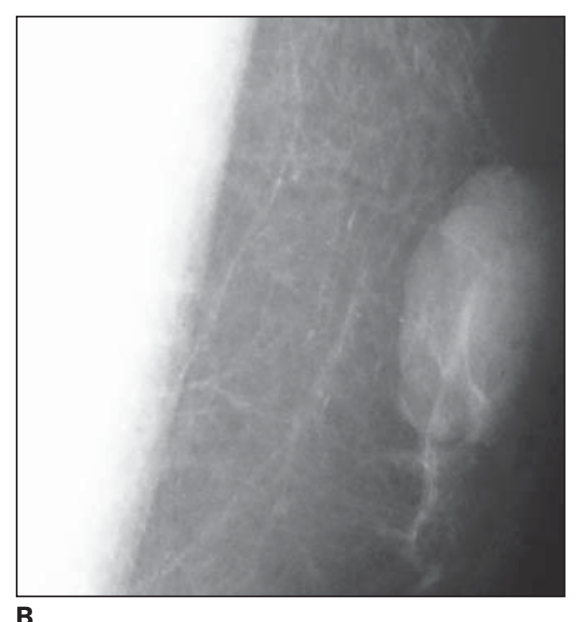

B 


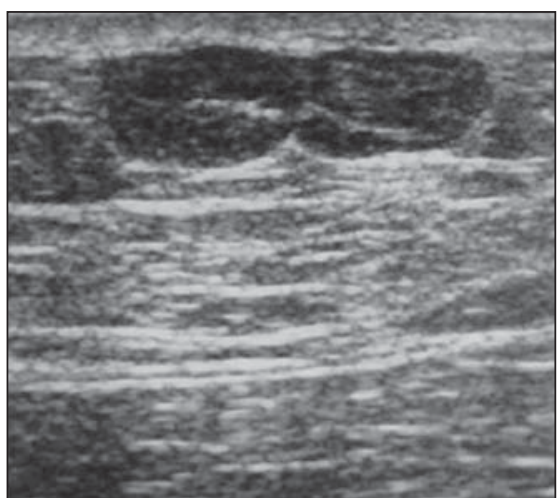

Figura 2. Ultra-sonografia mostra imagem hipoecogênica, elipsóide, contornos macrolobulados, com fina cápsula ecogênica e discreto reforço acústico posterior, medindo cerca de 2,6 $\mathrm{cm}$ de diâmetro no seu maior eixo, longitudinal em orientação à pele, correspondendo à imagem vista na mamografia.
Foi realizada excisão cirúrgica do tumor, cujo exame anatomopatológico exibiu nódulo com lobulações e de coloração pardacenta, medindo cerca de $2,0 \mathrm{~cm}$, e com conteúdo interno sólido-gelatinoso mostrando cisto folicular (Figura 3).

As lâminas da histopatologia revelaram estrutura intradérmica com abundância de epitélio escamoso queratinizado com estrutura pilosa associada (Figura 4), preenchendo os critérios histológicos do HFC, segundo Yamamoto et al. ${ }^{(\mathbf{3})}$ (Tabela 1).

\section{DISCUSSÃO}

O HFC é um hamartoma distinto formado por elementos estromais e epiteliais que não deve ser confundido, histopatologicamente, com hiperplasia de glândula sebácea, esteatocitoma, cisto dermóide da pele ou tricufoliculoma sebáceo ${ }^{(1-3)}$. O termo cístico refere-se a uma dilatação cística-símile do infundíbulo folicular. Ele não pode ser reconhecido clinicamente e depende do diagnóstico histopatológico ${ }^{(4)}$.

Sua manifestação comum ao exame físico é a de lesão cor de carne séssil ou de pápula pedunculada, mais comumente localizada na face, geralmente próxima ao nariz ${ }^{(\mathbf{1})}$. Não há relatos desta lesão estar em localização intra-epidérmica, como no nosso caso, simulando nódulo mamário.

Histologicamente, a mama masculina normal é composta de ductos subareolares

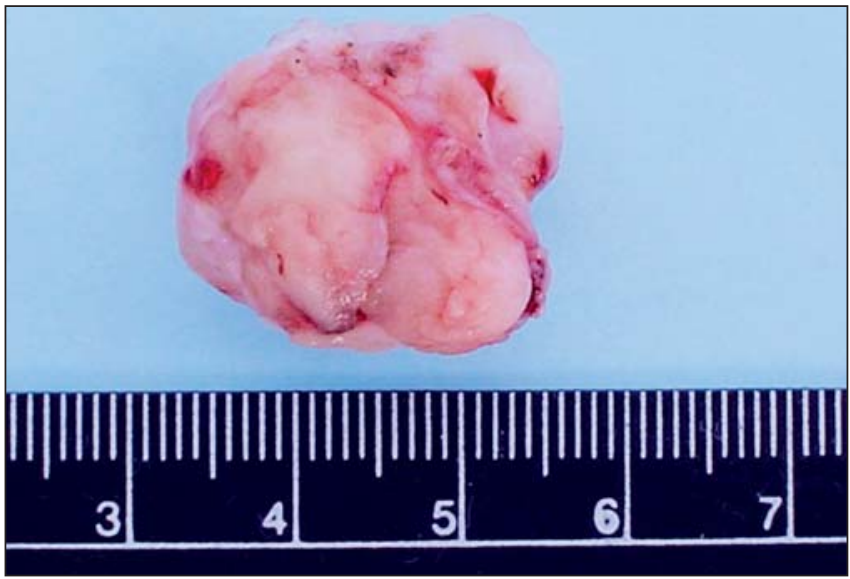

A

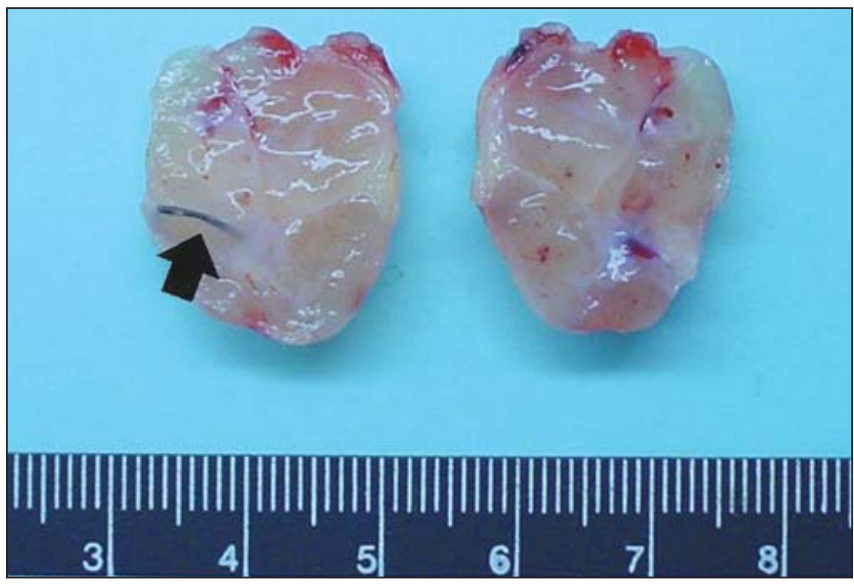

B

Figura 3. A: Nódulo com superfície externa de cor pardacenta, cruenta, medindo $2,0 \times 2,0 \mathrm{~cm}$, de consistência firme-elástica. B: Corte da lesão revela superfície interna com cor esbranquiçada brilhante e fasciculada. Nota-se também estrutura linear negra representando cisto folicular (seta).

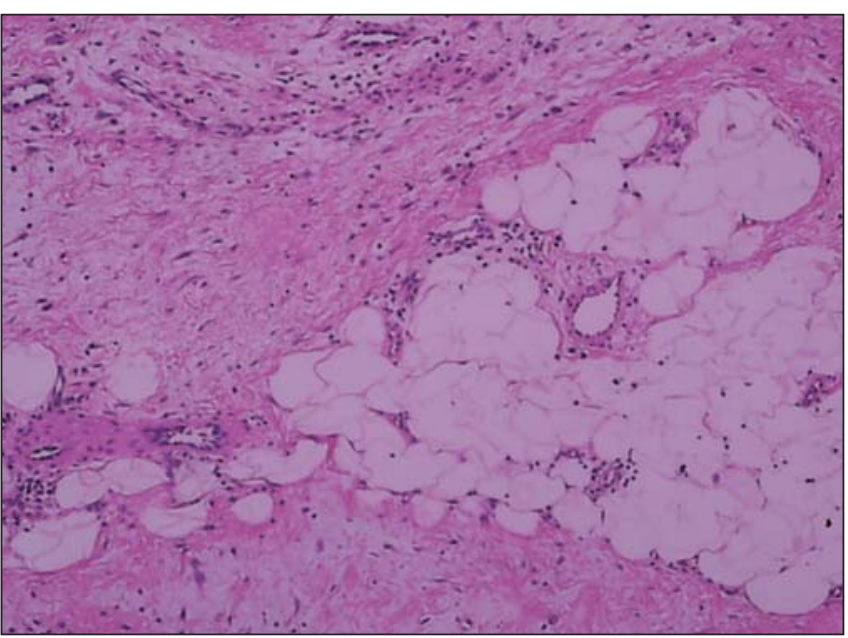

A

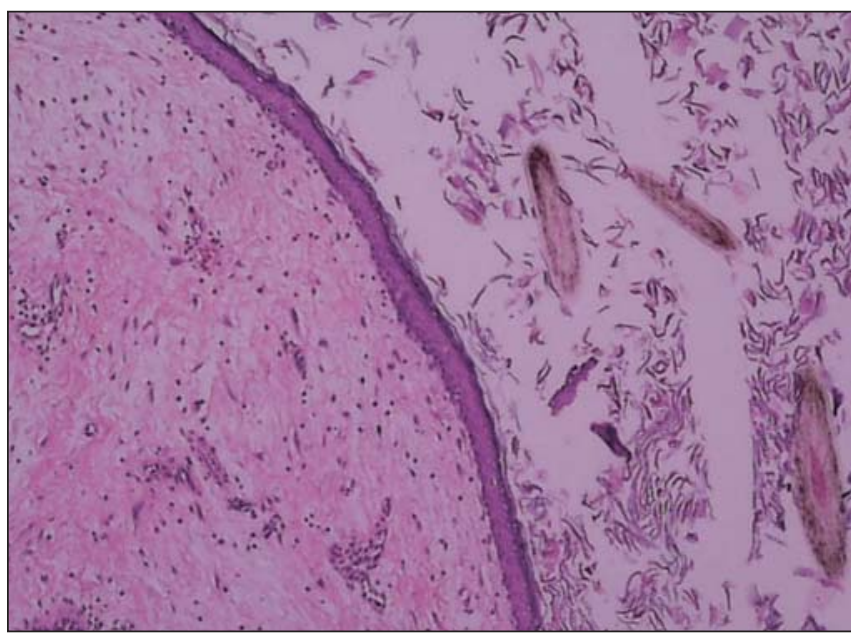

Figura 4. A: Corte histológico corado por hematoxilina-eosina mostra detalhe de tecido gorduroso no interior do hamartoma envolto por tecido mixóide (pequeno aumento). B: Outra área evidencia detalhes dos pêlos no tecido tumoral envoltos por material queratinizado, que também circunda epitélio limitando tecido mixóide na parte inferior esquerda da figura. 
Tabela 1 Características histológicas do hamartoma folículo-sebáceo cístico.

1. Estrutura cística intradérmica enfileirada por epitélio escamoso, que é similar à epiderme e ao epitélio infundibular

2. Numerosos nódulos sebáceos radiais à estrutura cística

3. Presença ocasional de estrutura pilosa madura ou rudimentar ou de glândulas apócrinas

4. Estroma em íntima relação com mudanças mesenquimais, variabilidade fibrosa, adiposa, vascular e tecido neural

Fonte: Yamamoto et al. ${ }^{(3)}$.

similares àqueles encontrados em meninas pré-púberes ${ }^{(\mathbf{5 , 6})}$. Assim, normalmente, todas as anormalidades encontradas na mama do homem são baseadas na etiopatogenia ductal. Quando estimulados, os ductos podem alongar-se e ramificar-se, dando origem, usualmente, à ginecomastia lesão mais comum neste tipo de mama ${ }^{(\mathbf{1 , 7}, \mathbf{8})}$. Alguns fatores podem ser apontados como causais de tais alterações, incluindo estrogênios endógenos e exógenos ou substâncias com atividade estrogênica-símile (hormônio adrenocortical, digitálico e espironolactona $)^{(\mathbf{1 , 3 , 7 , 8})}$, cirrose alcoólica e carcinoma hepatocelular ${ }^{(\mathbf{5})}$.

\section{Imagem da mama masculina}

A mamografia é o exame inicial de escolha para o homem com alteração mamária. O protocolo de radiografias em crâniocaudal e médio-lateral oblíqua deve ser substituído, nos homens, por uma única incidência caudo-cranial (ou crânio-caudal invertida), que mostra de maneira mais eficaz a mama afetada. Se o aumento for unilateral será difícil fazer o posicionamento da mama contralateral normal. A limitação fica restrita a pacientes com grande aumento abdominal por ascite ou obesidade ${ }^{(\mathbf{5})}$.

$\mathrm{O}$ aspecto mamográfico normal da mama masculina é de imagem radioluzente homogênea, determinada pela gordura, com algum tecido conectivo interlobular e ductos se estendendo do mamilo, sem os ligamentos de Cooper ${ }^{(\mathbf{7 , 9})}$.

Há considerável sobreposição de aspectos de imagens de lesões benignas e malignas na mama masculina ${ }^{(\mathbf{9})}$

$\mathrm{O}$ câncer de mama masculina é raro, representando menos de $1 \%$ dos casos de câncer de mama e $1 \%$ a $1,5 \%$ dos cânceres em homens ${ }^{(\mathbf{5 , 7 - 1 0})}$.

Os achados de imagem são inespecíficos e menos claros do que em mulheres. Normalmente, são massas pequenas, ovaladas ou lobuladas, com contornos bem definidos, indefinidos ou, mais incomumente, espiculados e de localização retroareolar. Microcalcificações são raras em homens e calcificações grosseiras podem ser vistam em lesões benignas e malignas. Pode haver queixa de dor ou desconforto leve $\mathrm{e}^{(\mathbf{9 - 1 1})}$. Ultra-sonograficamente, os achados são de lesões hipoecóicas e heterogêneas localizadas na região subareolar, excêntricas ao mamilo. Normalmente, têm margens irregulares e indistintas com variação acústica posterior (desde sombra até reforço do feixe sonoro) ${ }^{(\mathbf{1 1})}$.

\section{CONCLUSÃO}

Lesões nodulares mamárias têm uma gama de diagnósticos diferenciais e etiolo- gia nos quais os achados de imagem se sobrepõem e não definem critérios claros de benignidade e malignidade.

A comunidade médica especializada deve estar familiarizada com os achados normais e patológicos da mama masculina e estar apta ao diagnóstico precoce, para que o paciente possa dispor de melhores resultados estético e prognóstico.

\section{Agradecimento}

À Dra. Eliane Regina Bueno Garcia.

\section{REFERÊNCIAS}

1. Templeton SF. Folliculosebaceous cystic hamartoma: a clinical pathologic study. J Am Acad Dermatol 1996;34:77-81.

2. Kimura T, Miyazawa H, Aoyagi T, Ackerman AB Folliculosebaceous cystic hamartoma. A distinctive malformation of the skin. Am J Dermatopathol 1991;13:213-220.

3. Yamamoto O, Suenaga Y, Bhawan J. Giant folliculosebaceous cystic hamartoma. J Cutan Pathol 1994;21:170-172.

4. Ramdial PK, Chrystal V, Madaree A. Folliculosebaceous cystic hamartoma. Pathology 1998;30: 212-214.

5. Chantra PK, So GJ, Wollman JS, Bassett LW. Mammography of the male breast. AJR 1995;164:853858 .

6. Kopans DB. Mama masculina. In: Kopans DB, editor. Imagem da mama. $2^{a}$ ed. Rio de Janeiro, RJ: Medsi, 2000;497-502.

7. Michels LG, Gold RH, Arndt RD. Radiography of gynecomastia and other disorders of the male breast. Radiology 1977;122:117-122.

8. Dehner LP, Hill DA, Deschryver K. Pathology of the breast in children, adolescents, and young adults. Semin Diagn Pathol 1999;16:235-247.

9. Appelbaum AH, Evans GFF, Levy KR, Amirkhan RH, Schumpert TD. Mammographic appearances of male breast disease. RadioGraphics 1999;19: 559-568.

10. Ouimet-Oliva D, Hebert G, Ladouceur J. Radiographic characteristics of male breast cancer. Radiology 1978;129:37-40.

11. Yang WT, Whitman GJ, Yuen EHY, Tse GM, Stelling CB. Sonographic features of primary breast cancer in men. AJR 2001;176:413-416. 